\title{
Efeitos da ginástica abdominal hipopressiva sobre a musculatura pélvica em mulheres incontinentes
}

\author{
Effects of abdominal hipopressive gymnastics on the \\ pelvic muscle in incontinent women
}

Maiara Guerra Valente, ${ }^{1}$ Ariane Bôlla Freire, ${ }^{1}$ Amanda Albiero Real, ${ }^{1}$ Nathália Mezadri Pozzebon, ${ }^{1}$ Melissa Medeiros Braz, ${ }^{1}$ Patricia Xavier Hommerding ${ }^{1}$

Universidade Federal de Santa Maria (UFSM), Santa Maria, RS, Brasil.

Recebido em: Agosto 2015 / Aceito em: Dezembro 2015

melissabraz@hotmail.com

\section{RESUMO}

A incontinência urinária é definida pela Sociedade Internacional de Continência como a perda involuntária de urina. É uma condição multifatorial, que acomete ambos os sexos, em diversas idades. O envelhecimento, a multiparidade e cirurgias prévias são alguns dos fatores que levam à disfunção da musculatura perineal. Objetivo: avaliar os efeitos da ginástica abdominal hipopressiva sobre a força dos músculos do assoalho pélvico em um grupo de seis mulheres com incontinência urinária de esforço. Método: as participantes foram submetidas à ficha de avaliação, testes de força perineal e teste de ativação do músculo transverso do abdômen, pré e pós-tratamento. Foram realizadas 10 sessões fisioterapêuticas. Ao final do tratamento, as pacientes foram reavaliadas e os dados foram analisados pela estatística descritiva. Resultados: observou-se melhora na força do assoalho pélvico, bem como melhora na ativação do transverso abdominal. Considerações finais: o protocolo da ginástica abdominal hipopressiva parece ser um método eficiente para auxiliar mulheres com incontinência urinária de esforço, pois aumentou a força perineal e ativou o músculo transverso do abdômen nas participantes deste estudo.

Palavras-chave: Incontinência urinária de esforço; Fisioterapia; Ginástica hipopressiva.

\section{ABSTRACT}

Urinary incontinence is defined by the International Continence Society as the involuntary loss of urine. It is a multifactorial condition that affects both sexes at different ages. Aging, multiparity, previous surgeries, are some of the factors that lead to perineal muscles dysfunction. Objective: to evaluate the effects of Abdominal Hipopressive Gymnastics on the strength of pelvic floor muscles in a group of six women with stress urinary incontinence. Method: participants underwent evaluation form, performed strength tests and perineal test of transversus abdominis muscle activation test, pre and post treatment. The research period held a total of 10 physical therapy sessions. At the end of the treatment the patients were evaluated and the data were analyzed using descriptive statistics. Results: there was significant improvement in the pelvic floor strenght, as well as improvement in the transversus abdominis activation. Closing remarks: the protocol of abdominal hipopressive gymnastics was an effective method to help women with stress urinary incontinence, as increased perineal strength and activated the transversus abdominis muscle in participants of this study.

Keywords: Stress urinary incontinence, Physiotherapy, Hipopressive gymnastics.

\section{INTRODUÇ̃̃O}

A incontinência urinária (IU) é definida como a perda involuntária de urina, constituindo-se em problema social ou de higiene. ${ }^{1,2}$ Ela caracteriza-se como uma condição multifatorial que acomete homens e mulheres em diferentes faixas etárias, com prevalência em mulheres, devido a uretra feminina ser relativamente curta $(3$ a $4 \mathrm{~cm}$ ), além de razões vasculares e tróficas. Cerca de $60 \%$ das mulheres acima de 60 anos apresentam IU. ${ }^{3}$ 
Os danos causados ao assoalho pélvico pela gestação e pelo parto, a idade avançada, cirurgias prévias, multiparidade, infecções do trato urinário, fragilidade da musculatura do assoalho pélvico e atrofia tecidual levam à disfunção da musculatura perineal, que tende a se agravar após a menopausa. ${ }^{4,5}$ Essas disfunções da musculatura perineal podem estar associadas à IU, que é classificada em três tipos: esforço (IUE), urgência (IUU) e mista (IUM). ${ }^{6}$

O tipo mais frequente é a incontinência urinária de esforço, conceituada pela perda involuntária de urina sem a contração do músculo detrusor e ocorre quando a pressão intravesical ultrapassa a pressão na luz da uretra, através de uma falha do seu mecanismo de oclusão. ${ }^{7}$ Ela acontece quando há um aumento da pressão intra-abdominal, como no exercício físico, tosse ou espirro, que pode predispor à fraqueza perineal. ${ }^{1}$

O períneo é uma região anatômica formada pelo diafragma pélvico e o diafragma urogenital, que têm como função o controle da micção, da defecação, dos esfíncteres vesicais e anais, durante tosse e espirro, além de sustentar as vísceras intra-pélvicas. ${ }^{8}$ Dessa forma, a musculatura do assoalho pélvico assume papel relevante no mecanismo da continência urinária, pois é fundamental na manutenção do suporte anatômico e da pressão intra-uretral. ${ }^{5}$

Problemas psicológicos e emocionais também estão associados à IU. Sentimentos como vergonha, medo, nervosismo e depressão geralmente são relacionados ao forte odor causado pela perda de urina. A mulher incontinente raramente fala sobre o seu problema e, por acreditar não ter cura, muitas sofrem em silêncio. ${ }^{9}$

A Fisioterapia, atualmente, é considerada um tratamento de primeira linha para a IU, apresentando resultados vantajosos em relação à cirurgia, por ser conservadora, pouco invasiva, com baixo índice de efeitos colaterais e custo reduzido. Técnicas cada vez menos invasivas vêm sendo desenvolvidas nos últimos anos, dentre elas encontra-se a Ginástica Abdominal Hipopressiva (GAH). ${ }^{10}$

A GAH é um conjunto de posturas associadas a movimentos respiratórios que provocam uma queda na pressão intra-abdominal, ativação sinérgica de músculos do pavimento pélvico e músculos abdominais, especialmente o transverso abdominal. A ativação desse músculo pode coativar a musculatura perineal. Em longo prazo, leva ao aumento no tônus do assoalho pélvico e músculos abdominais, diminuindo significativamente o risco de perda urinária. ${ }^{12}$ Além disso, a GAH pode ser definida como uma técnica postural, pois realiza uma ativação dos diferentes grupos musculoesqueléticos, que são antagonistas do diafragma do ponto de vista postural, podendo assim causar uma diminuição na pressão intra-torácica e intra-abdominal. ${ }^{12}$

Diante do exposto acima, essa pesquisa visou investigar os efeitos de um programa de ginástica hipopressiva, sobre a incontinência urinária de esforço.

\section{MÉTODO}

Foi realizada uma pesquisa do tipo semi experimental, sem grupo controle. A população foi composta por mulheres com queixa de IUE. Foram incluídas mulheres, com idades variando de 55 a 70 anos, no período pós-menopausal. Como critérios de exclusão constataram-se mulheres: nulíparas, diabéticas, hipertensas, com problemas respiratórios ou neurológicos, que realizassem tratamento fisioterapêutico e/ou medicamentoso para a incontinência urinária ou terapia de reposição hormonal.

A amostra foi composta, inicialmente, por $10 \mathrm{mu}$ Iheres; porém, de acordo com os critérios de exclusão, 6 compuseram a amostra final, de forma não probabilística acidental. A coleta de dados teve início somente após a autorização do local de coleta de dados e a aprovação do Comitê de Ética e Pesquisa da Instituição, sob o número do protocolo 269.2011.2.

Primeiramente, as mulheres foram convidadas a participar da pesquisa por meio de contato telefônico, encontrado através das listas de espera de atendimento do Sistema Integrado de Saúde, do Centro Universitário Franciscano (UNIFRA). Foram explicados os objetivos da pesquisa e as mulheres foram convidadas a participar mediante a assinatura do termo de consentimento livre e esclarecido (TCLE), sendo o sigilo garantido pelo termo de confidencialidade.

$\mathrm{Na}$ primeira sessão, foi aplicada a ficha de avaliação, contendo identificação da paciente, anamnese, exame físico, história uroginecológica e obstétrica da mulher. ${ }^{3}$ Posteriormente, foi realizado o exame perineal, conforme a descrição que segue: a paciente foi colocada em posição ginecológica, enquanto a pesquisadora, calçando luvas descartáveis, realizou inspeção perineal com o objetivo de observar a vulva, a pele, presença de cicatrizes, analisar a mucosa vaginal, corrimento sinais de atrofia pelo hipoestrogenismo. ${ }^{12}$ Caso houvesse corrimento ou sinais de infecção, seria encaminhada para acompanhamento médico.

Após a inspeção perineal, foi realizado um teste, através da inserção bidigital no canal vaginal da paciente, para verificar o grau da força perineal, graduada pela escala de Ortiz. A paciente permaneceu na mesma posição, enquanto a pesquisadora, usando luvas de procedimento, utilizou o $2^{\circ}$ e $3^{\circ}$ dedos para afastar os pequenos lábios com uma de suas mãos e com a outra realizou uma introdução bidigital na vagina, sendo a paciente orientada a executar uma contração do assoalho pélvico e manter o máximo de tempo que conseguisse. Dessa forma, avaliou-se a capacidade de contração dessa musculatura e o tempo de duração da mesma.

Em seguida, foi realizada a avaliação da força perineal através do perineômetro da marca Quark. Previamente a sua introdução na vagina da voluntária, a sonda foi recoberta por um preservativo lubrificado, o que permitiu respeitar o controle da assepsia e evitar a contaminação de uma paciente para outra. Somente depois de a sonda estar recoberta pelo preservativo, efetuou-se a introdução vaginal, com a finalidade de mensurar, em mililitros de mercúrio $(\mathrm{mmHg})$, a força da musculatura do assoalho pélvico. A paciente permaneceu na posição ginecológica, com os pés apoiados na maca e foi solicitado que contraísse a musculatura perineal, acompanhando no visor do aparelho o sinal luminoso que deu o retorno visual da sua contração máxima. Foram realizadas três contrações máximas com comando verbal (com intervalo de 5 segundos entre elas) da pesquisadora e registrado o maior valor alcançado. ${ }^{3}$ 
Por último, foi avaliada a ativação do transverso do abdome, através do esfigmomanômetro. A paciente foi posicionada em decúbito dorsal e Ihe foi ensinado a ativar a musculatura profunda abdominal com instruções verbais, como encolher o abdome, levando o umbigo em direção à coluna no tempo expiratório. A investigada assumiu a posição neutra da coluna e tentou mantê-la, enquanto encolheu suavemente e deprimiu os músculos abdominais. Para iniciar o teste, a pesquisada assumiu a posição decúbito ventral sobre a unidade de biofeedback de pressão. O esfigmomanômetro foi nivelado ao centro da parede abdominal. $\mathrm{O}$ manômetro foi insuflado até $70 \mathrm{mmHg}$, sendo instruído a puxar para dentro o abdome para ter uma ativação total da musculatura abdominal, incluindo transverso do abdome. Assim, o aparelho mediu em qual nível de pressão a paciente conseguiu manter a contração. O resultado satisfatório foi avaliado quando a pressão era mantida entre 64 e 70 $\mathrm{mmHg}$ e insatisfatório quando a pressão era abaixo deste nível. ${ }^{14}$

Após a avaliação, foram realizadas duas sessões fisioterapêuticas semanais em grupo, durante quatro semanas, totalizando dez sessões, com duração de 50 minutos cada, ${ }^{11}$ sendo que a primeira e a última sessão foram destinadas à avaliação e reavaliação das pacientes. Para as sessões, foi utilizado um programa de ginástica hipopressiva adaptado de Teixeira et al. ${ }^{15}$

As pacientes realizaram os exercícios seguindo a orientação da pesquisadora, a qual estava ao seu lado, demonstrando os mesmos. Foram executadas seis posturas realizadas em um ritmo muito lento, as quais eram mantidas, variando de 5 a 20 segundos, repetidas 3 vezes, realizando um período de descanso de 20 segundos entre cada exercício e, devido à grande concentração na realização das posturas, uma parte deste tempo foi realizada em apnéia. Os exercícios visaram uma tonificação abdominal e perineal. ${ }^{11}$ Para o início da sessão foi realizado o treino da respiração: deve-se inspirar, em seguida solta-se o ar pela boca, contraindo o abdômen (expiração); e no final da expiração, bloqueia-se o ar, as costelas irão se afastar e o abdômen ficará contraído; deve-se manter o ar bloqueado o maior tempo possível. A graduação dos exercícios foi às diferentes posições realizadas: em pé, de joelhos, sentada, quatro apoios, cócoras, deitada, além do tempo de apneia, o qual foi aumentando conforme o limite de cada paciente.

No final do tratamento, as pacientes foram reavaliadas e os dados foram analisados pela estatística descritiva.

\section{RESULTADOS}

Foram estudadas seis pacientes do sexo feminino, com idades variando de 55 a 70 anos, com média de 63,3 anos de idade. A queixa principal das mulheres referia-se a incontinência urinária de esforço. Todas apresentavam no mínimo dois fatores de risco como a multiparidade e a diminuição do colágeno e estrogênio associado à menopausa. Tossir foi a atividade que todas referiram perder mais urina. Sobre a quantidade de perda, quatro delas relataram perda de urina em gotas, uma em jatos e uma com perda contínua.

Para quatro das mulheres, os sintomas iniciaram-se de um a quatro anos, para uma a menos de um ano e uma de 8 a 12 anos. Quatro delas relatam que os sintomas estão iguais desde que começaram e duas relatam piora nos sintomas. Quanto o uso de forro, três usam e três não. A realização de cirurgia ginecológica para tratamento da incontinência urinária foi citada apenas por uma, não havendo melhora no quadro.

O tratamento de reposição hormonal foi realizado por três delas. Quatro ainda possuem atividade sexual ativa, sendo que cinco consideram sua vida sexual boa e uma considera ótima.

Na primeira avaliação, quando se realizou o teste de força perineal segundo a Escala de Ortiz, quatro das mulheres apresentaram grau 3 e duas grau 2. Já, na segunda avaliação, todas apresentaram grau 4.

$\mathrm{Na}$ avaliação da força em $\mathrm{mmHg}$, utilizando o perineomêtro, duas alcançaram $8,0 \mathrm{mmHg}$, duas alcançaram 4,4 mmHg, uma alcançou 4,8 mmHg e uma alcançou 12,8 mmHg. Na reavaliação quatro apresentaram força máxima $(46,4 \mathrm{mmHg})$ e as outras duas apresentaram 38,4 $\mathrm{mmHg}$.

Quando avaliada a ativação do transverso do abdome, em $\mathrm{mmHg}$, através do esfigmomanômetro, $100 \%$ das mulheres não conseguiram sustentar a contração acima de $64 \mathrm{mmHg}$, então a força foi avaliada como insatisfatória. Na segunda avaliação, todas obtiveram força satisfatória.

Os dados relativos à avaliação funcional do assoaIho pélvico são apresentados na tabela I.

A avaliação subjetiva dos resultados demonstrou que as seis mulheres consideraram-se satisfeitas com o tratamento fisioterapêutico e que o recomendariam para outras mulheres. Além disso, gostariam que o tratamento tivesse continuidade.

Observou-se que a falta de conhecimento do grupo estudado sobre a existência e atuação da fisioterapia, nesta área, colaborou para o retardo na procura de tratamento.

Tabela 1 - Avaliação da força perineal (Ortiz, Perina) e do transverso abdominal antes e após ginástica hipopressiva.

\begin{tabular}{|c|c|c|c|c|c|c|}
\hline \multirow[t]{2}{*}{ Pacientes } & \multicolumn{2}{|c|}{ Ortiz } & \multicolumn{2}{|c|}{ Perina $(\mathrm{mmHg})$} & \multicolumn{2}{|c|}{ Transverso Abdominal } \\
\hline & Pré & Pós & Pré & Pós & Pré & Pós \\
\hline A & 3 & 4 & 4,8 & 46,4 & Insatisfatório & Satisfatório \\
\hline B & 2 & 4 & 4,4 & 38,4 & Insatisfatório & Satisfatório \\
\hline C & 2 & 4 & 4,4 & 38,4 & Insatisfatório & Satisfatório \\
\hline D & 3 & 4 & 12,8 & 46,4 & Insatisfatório & Satisfatório \\
\hline $\mathbf{E}$ & 3 & 4 & 8,0 & 46,4 & Insatisfatório & Satisfatório \\
\hline $\mathbf{F}$ & 3 & 4 & 8,0 & 46,4 & Insatisfatório & Satisfatório \\
\hline
\end{tabular}




\section{DISCUS5ÃO}

Estudos demonstram que a IU pode acometer ambos os sexos, mas principalmente as mulheres em diversas fases da sua vida, sendo a prevalência maior em idosas. ${ }^{16}$

As mulheres pesquisadas nesse estudo apresentaram no mínimo dois fatores de risco para incontinência urinária. Inúmeros são os fatores de risco que se associam e contribuem para o aparecimento de IU, incluindo o parto vaginal que causa trauma e desnervação do assoalho pélvico, atividades que aumentem a pressão intra-abdominal como o suporte de peso excessivo, tosse crônica, constipação, obesidade, gravidez, multiparidade, envelhecimento natural das fibras musculares, diminuição do colágeno e estrogênio associado com a menopausa, entre outros. ${ }^{17,18}$

Neste estudo todas as voluntárias estavam no período pós-menopausal e apresentavam déficit na força perineal, o que corrobora com a citação de Mourão ${ }^{19}$, na qual o envelhecimento, as taxas de hormônio decaem, a espessura dos músculos do assoalho pélvico parece diminuir, com isso ocorre um decréscimo na força muscular, sendo a menopausa o ponto culminante a partir do qual os músculos do assoalho pélvico enfraquecem muito rapidamente.

A Sociedade Internacional de Continência, em 2005, recomendou a fisioterapia como primeira opção de tratamento para IU, pois dispõe de diversas técnicas e recursos, apresenta baixo custo e risco, além da eficácia comprovada, ${ }^{17}$ obtendo efeitos positivos, como a diminuição de prejuízos cirúrgicos, restauração efetiva da função e propriocepção da musculatura do períneo e melhora da IU através da aplicação de diferentes modalidades e entre elas destaca-se a ginástica hipopressiva. ${ }^{15}$ Os resultados do presente estudo com ginástica hipopressiva mostraram melhora significativa da IU. Houve aumento da força perineal bem como melhora da ativação do músculo transverso do abdômen. No que se refere à força perineal, após o tratamento $100 \%$ das pacientes apresentaram melhora, sendo que 66,6\% apresentaram força máxima no perineômetro e 100\% força máxima no teste de Ortiz. Tal achado corrobora com o trabalho de Teixeira et al., ${ }^{15}$ o qual cita que a técnica da GAH atua, tanto na prevenção quanto na cura, incluindo a alteração na distribuição de pressões, estimulando e fortalecendo as paredes da vagina e a musculatura perineal. Além disso, aumenta o tônus de base do períneo, ajuda a posicionar os órgãos do baixo ventre e favorece a correção postural.

Outra pesquisa também objetivou avaliar o grau de força muscular do assoalho pélvico em um grupo de mulheres com IUE e reavaliá-lo após aplicação de um protocolo com biofeedback. Houve melhora significativa quanto à força de contração do assoalho pélvico, em relação à atividade eletromiográfica em microvolts $(p=0,0117) .^{20}$

Esta pesquisa utilizou um protocolo de ginástica hipopressiva no solo, enquanto que, um segundo estudo de Caufriez et al., ${ }^{21}$ comparou a eficácia da GAH dentro e fora d'água, concluindo que realizar $\mathrm{GAH}$ em meio líquido não oferece nenhuma vantagem significativa em termos de pressão abdominal. No entanto, o efeito gerado pela pressão hidrostática melhora a mo- bilidade das cúpulas diafragmáticas. O presente estudo avaliou a eficácia da GAH sobre a ativação do músculo transverso do abdome, no qual obteve melhora. O que condiz com o autor Palma, ${ }^{11}$ em que a GAH realiza uma ativação sinérgica de músculos do pavimento pélvico e músculos abdominais, especialmente o músculo transverso abdominal.

\section{CONSIDERACִÕES FINAIS}

Pode-se afirmar que este estudo, através da aplicação de um protocolo com ginástica abdominal hipopressiva, obteve bons resultados em relação à força perineal e ativação do músculo transverso do abdômen em mulheres com incontinência urinária de esforço. Pesquisas futuras, com uma coorte maior de pacientes, que possam avaliar os efeitos terapêuticos à longo prazo deverão ser consideradas.

\section{REFERÊNCIAS}

1. Rett MT, Simões JA, Herrman V, Gurgel MSC, Moraes SS. Qualidade de vida em mulheres após tratamento da incontinência urinária de esforço com fisioterapia. Rev Bra Ginecol Obstet 2007;29(3):134-40.

2. Polden M, Mantle J. Fisioterapia Obstetrícia E Ginecologia. 2. Ed. São Paulo: Santos, 2002.

3. Moreno AL. Fisioterapia em uroginecologia. 2. Ed. São Paulo: Manole, 2009.

4. Oliveira KAC, Rodrigues ABC, Paula AB. Técnicas fisioterapêuticas no tratamento e prevenção da incontinência urinária de esforço na mulher. Rev Eletrôn F@Pciência, Apucarana/PR 2007;1(1):31-40.

5. Santos AL, Oliveira DDS; Bianchi FR, de Lima APB, Castilho JD, Lucato Junior RV, et al. Intervenção fisioterapêutica na incontinência urinária de esforço em mulheres, com ênfase no tratamento postural e comportamental. Rev Unorp 2011;5(12):25-47. Disponível em: <http://Www.Unorp.Br/ Asp/..\%5crevista\%5csaudeiii\%5c3.Pdf.> Acesso Em: 17 Jun 2011.

6. Caldas CP, Conceição IRS, José RMDC, Silva BMC. Terapia comportamental para incontinência urinária da mulher idosa: uma ação do enfermeiro. Texto Contexto Enfermagem 2010; 19(4):783-8

7. Auge AP, Zucchi CM, Costa FMP, Nunes K, Cunha, LPDM, Silva PF, et al. Comparações entre os índices de qualidade de vida em mulheres com incontinência urinária submetidas ou não ao tratamento cirúrgico. Rev Bras Ginecol Obstet 2006; 28(6):352-7.

8. Nolasco J, Martins L, Berquo M, Sandoval RA. Atuação da cinesioterapia no fortalecimento muscular do assoalho pélvico feminino: revisão bibliográfica. Rev Dig 2008;12(117). Disponível em: <http://Www.Efdeportes.Com/Efd117/ Fortalecimento-Muscular-Do-Assoalho-Pelvico-Feminino. Htm.> Acesso Em: 16 Jun 2011.

9. Lopes MHBDM, Higa R. Restrições causadas pela incontinência urinária à vida da mulher. Rev Esc Enf 2006; 40(1):34-41.

10. Rodrigues MHR. Fisioterapia no tratamento da incontinência urinária de esforço pós-prostatectomia radical: revisão da literatura. Soc Urol Contemp 2010;19(4):20-30.

11. Palma PCR. Urofisioterapia: aplicações clínicas das técnicas 
fisioterapêuticas nas disfunções miccionais e do assoalho pélvico. Campinas: Personal Link Comunicações, 2009.

12. Caufriez M, Fernánde JC, Fanzel R, Snoeck T. Efectos de un programa de entrenamiento estructurado de Gimnasia Abdominal Hipopresiva sobre La estática vertebral cervical y dorsolumbar. Fisioterapia 200;28(4):205-16.

13. Etienne MDA, Waitman MC. Disfunções Sexuais Femininas: A Fisioterapia Como Recurso Terapêutico. São Paulo: Livraria Médica Paulista, 2006.

14. Frigo L, Braz MM. Avaliação da ativação muscular do centro de força de mulheres nuligéstas. Trabalho final de graduação. Centro Universitário Franciscano, Curso de Fisioterapia, Santa Maria, 2009.

15. Teixeira A, Guimarães CA, Zimer SDRA, Oliveira APMD. Tratamento fisioterapêutico na incontinência urinária de esforço em mulheres de 35 a 55 anos. Rubs, Curitiba 2005; 1(3):12-16.

16. Baracho SM. Preditores de incontinência urinária de esforço em mulheres primíparas que realizaram parto vaginal. Dissertação De Mestrado. Belo Horizonte: Escola De Educação Física, Fisioterapia E Terapia Ocupacional, Universidade Federal De Minas Gerais, 2010.

17. Sousa JG, Ferreira VR, Oliveira RJD, Cestari CE. Avaliação da força muscular do assoalho pélvico em idosas com incontinência urinária. Fisiot Mov 2011;24(1):39-46

18. Correia GN. Efeitos de um protocolo de fortalecimento da musculatura do assoalho pélvico em grupo para mulheres com perda urinária. Dissertação De Mestrado. Universidade Federal De São Carlos. Centro De Ciências Biológicas E Da Saúde. Programa De Pós-Graduação Em Fisioterapia, São Carlos, 2010

19. Mourão CM, Pina RC, Wanderley TJ. Avaliação dos efeitos do tratamento do assoalho pélvico com utilização do miofeedback perina em mulheres. Trabalho De Conclusão De Curso. Universidade Da Amazônia. Curso De Fisioterapia. Belém, 2006.

20. Costa CHSD, Santos LPVD, Ferreira MDO. O fortalecimento dos músculos do assoalho pélvico com a técnica de biofeedback em mulheres com incontinência urinária de esforço. Trabalho de Conclusão de Curso. Universidade Da Amazônia, Centro De Ciências Biológicas E Da Saúde, Curso De Fisioterapia, Belém, 2008.

21. Caufriez M, Fernández JC, Guignel G, Heimann A. Comparación de lãs variaciones de presión abdominal en médio acuático y aéreo durante la realización de cuatro ejercicios abdominales hipopresivos. Rev Iber Fisiot Kines 2007;10(1):12-23 